Special issue of the 3rd International Conference on Computational and Experimental Science and Engineering (ICCESEN 2016)

\title{
Investigation of GSM, LTE and Wi-Fi Electromagnetic Radiation in Dwellings
}

\author{
Y. KARAN ${ }^{a *}$, N. As ${ }^{a}$ AND M.E. ŞAHIN ${ }^{b}$ \\ ${ }^{a}$ Department of Physics, Recep Tayyip Erdoğan University, Rize, Turkey \\ ${ }^{b}$ Department of Electrical and Electronics Engineering, Recep Tayyip Erdoğan University, Rize, Turkey

\begin{abstract}
Electromagnetic radiation exposure increases with the rise of the wireless communication devices. Electromagnetic radiation, especially near $2400 \mathrm{MHz}$ band, has gradually increased with the widespread of wireless fidelity adapter in indoor environment. In this study, electromagnetic radiation in dwellings where different Wi-Fi adapters and routers are used was assessed by measuring the electric field and compared with other electromagnetic radiation source and the limits set by the Information and Communication Technologies Authority. Measurements were taken in the range of $760-2690 \mathrm{MHz}$ bands used by GSM, 3G/LTE and Wi-Fi technologies. The detected values for all frequencies are significantly lower than the limits of the Information and Communication Technologies Authority. Among the electromagnetic radiation sources, electromagnetic radiation of Wi-Fi adapter is higher than GSM/3G/LTE sources radiation in dwellings. Electromagnetic radiation values in nearest point of high power adapter Wi-Fi antenna are close to the Information and Communication Technologies Authority limit value. Results show that electromagnetic radiation based on Wi-Fi adapters or routers can be higher than GSM based
\end{abstract} \\ electromagnetic radiation.
}

DOI: 10.12693/APhysPolA.132.509

PACS/topics: wireless network adapter, electromagnetic radiation, electromagnetic pollution, global system for mobile communications, wireless fidelity

\section{Introduction}

Exposure of electromagnetic (EM) radiation (EMR) is exponentially increased as ubiquitous of wireless devices and their daily usage time rise in our lives. Wireless networks and use of devices that emit EM field are spreading out by the time [1-4]. These developments cause electromagnetic pollution and environmental pollution. These pollutants may threaten human health. Furthermore, for some people suffering from electromagnetic hypersensitivity, electromagnetic field (EMF) pollution causes many more minor and severe health risks [5]. Scientists have identified EMR as an indoor and outdoor environmental pollutant. Therefore, different procedures or methods can be used to assess environmental exposure to $\mathrm{RF}$ radiation $[6,7]$.

There have been many studies about measurement of electromagnetic radiation. Some of these studies are made in urban areas, campus areas, and different dwellings [7-10]. The electric field strength of base stations and even $\Upsilon$ radiation dose levels were measured with the samples taken from different distances from a base station [10-14]. In addition, electromagnetic radiation measurement of a high power wireless network adapter and Wi-Fi access points are investigated in some studies $[15,16]$.

In this study, electric field strengths are measured in different dwellings. Measurements were taken in the range of $760-2690 \mathrm{MHz}$ bands used by GSM, 3G/LTE

*corresponding author; e-mail: yasin.karan@erdogan.edu.tr and $\mathrm{Wi}-\mathrm{Fi}$ technologies. The rest of this paper is organized as follows. In Sect. 2, electromagnetic environment, effects and guidelines are given. In Sect. 3, measurements and results are given. Finally, Sect. 4 contains conclusions.

\section{Electromagnetic environment}

Electromagnetic waves and sources, biological effects of electromagnetic fields, standard and guidelines are given in this chapter shortly. The details of this chapter are given in previous studies $[9,11]$.

\subsection{Electromagnetic waves and sources}

Radiation is the propagation process of energy of waves through a medium. Two types of radiations are ionized and non-ionized radiation. Non-ionized radiation consists of EM radiation. EM fields consist of electric and magnetic field components. Electric fields arise from voltage and are measured as volts per meter $(\mathrm{V} / \mathrm{m})$. Magnetic fields arise from current flows and measured as amperes per meter $(\mathrm{A} / \mathrm{m})$. Both electric and magnetic field components oscillate in phase perpendicular to each other.

Non-ionizing radiation spectrum covers the frequencies below $300 \mathrm{GHz}$ which are radio waves and other frequency bands such as microwaves, telecommunication signals, GSM base stations, radar signals, RF-TV waves, high voltage systems, power systems.

\subsection{Biological effects of electromagnetic fields}

Long-term exposure to EM radiation is thought to cause lasting effects. The most obvious effect of nonionizing radiation is temperature increase in the tissues. 
High frequency $(\mathrm{HF})$ or RF electromagnetic fields can heat all body or parts of the body depending on the frequency. The body and body parts act as a lossy antenna in the $0.75 \mathrm{~m}$ to $2 \mathrm{~m}$ wavelength range. The body qualitative absorption curve as a function of radio frequency is shown in Fig. 3 [17].

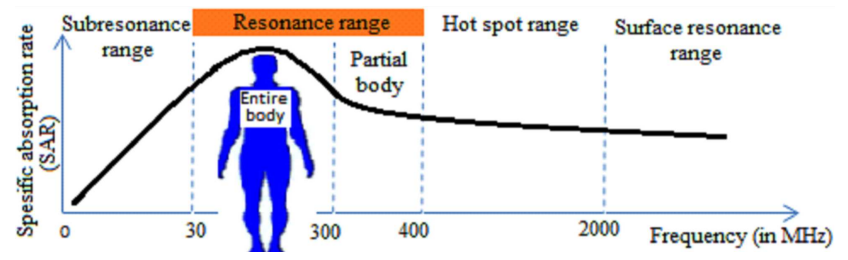

Fig. 1. The body absorption curve.

The absorbed electromagnetic wave energy by the body in living organisms is giving by specific absorption rate (SAR). SAR is the amount of energy per kg absorbed by a normal human body. The calculation of SAR value is simplified by Eq. (1):

$$
\mathrm{SAR}=\frac{\sigma E^{2}}{\rho}[\mathrm{W} / \mathrm{kg}],
$$

where $\sigma$ is conductivity $[\mathrm{S} / \mathrm{m}], E$ is the electric field $[\mathrm{V} / \mathrm{m}]$ and $\rho$ is density $[\mathrm{kg} / \mathrm{m}]$. SAR value is also interrelated with temperature as shown in Eq. (2):

$$
\mathrm{SAR}=c \frac{\mathrm{d} T}{\mathrm{~d} t}[\mathrm{~W} / \mathrm{kg}]
$$

where $c$ is specific heat capacity $[\mathrm{J} /(\mathrm{g} \mathrm{K})], T$ is the temperature and $t$ is time. The $\mathrm{SAR}=4 \mathrm{~W} / \mathrm{kg}$ is founded as the exposure value that causes $1^{\circ} \mathrm{C}$ to increase on the average mass of a human body temperature [19]. Limit values are defined by $\mathrm{SAR}$ value. SAR value is a parameter that cannot be measured directly. Therefore, electric and/or magnetic field strength in the near field and power density in the far field is measured to evaluate SAR. This specific absorption rate is RF electromagnetic energy absorbed by the body in average six minutes.

\subsection{Standard and guidelines}

The limit values about electromagnetic radiation determined by International Commission on Non-Ionizing Radiation Protection (ICNIRP) are the most widely accepted standard in many countries all around the world. ICNIRP is an independent research organization and officially recognized by the World Health Organization (WHO) and the International Labour Organization (ILO). ICNIRP guideline is the product of collaboration of universities and research institutions with many engineers, biologists, physicists, epidemiologists and other relevant inter-disciplinary team of scientists. The Information and Communication Technologies Authority (ICTA) prepared and published a regulation on the security certification of electronic communication devices in Turkey. Table I shows the limit values of ICNIRP and ICTA for electric field, magnetic field and power density $[19,20]$.
Reference levels for general public exposure.

\begin{tabular}{c|c|c|c|c}
\hline \hline \multirow{2}{*}{$\begin{array}{c}\text { Frequency } \\
\text { range [MHz] }\end{array}$} & \multicolumn{2}{|c|}{$E$-field [V/m] } & \multicolumn{2}{c}{$H$-field [A/m] } \\
\cline { 2 - 5 } & ICNIRP & ICTA & ICNIRP & ICTA \\
\hline $400-2000$ & $1.375 f^{1 / 2}$ & $0.305 f^{1 / 2}$ & $0.0037 f^{1 / 2}$ & $0.00082 f^{1 / 2}$ \\
900 & 41.25 & 9.15 & 0.111 & 0.0246 \\
1800 & 58.34 & 12.94 & 0.157 & 0.0348 \\
$2000-60000$ & 61 & 13.5 & 0.16 & 0.035
\end{tabular}

\section{Measurements and results}

Electromagnetic field measurements were realized with a compact spectrum analyzer device (SRM 3006, Narda Safety Test Solutions GmbH, Pfullingen, Germany). It measures the electric field in the $420 \mathrm{MHz}-6 \mathrm{GHz}$ frequency range with a triaxial coil antenna. Frequency band can be selected for different frequencies. The distance that splits the near and far field follows $2 D^{2} \lambda$ parameter. $R<3 \lambda$ is accepted as near field criterion and $\lambda<D$ and $R>2 D^{2} \lambda$ is accepted as far field limit, where $\lambda$ is wavelength, $D$ is diameter and $R$ is radius $[11,20]$. Measurement channels and measurement results for three dwellings are given in Table II.

Using this maximum and maximum average electric field strength (EFS) measurement results for three dwellings in Table II, the graphics are obtained as in Fig. 2a-c. The graphics show electric field strength for different channels. Among the average maximum EFS values for all the channels, WLAN channel shows the highest EFS value in the all dwellings. For the instant measurements, any channels peak values may show highest EFS, depending on the distance between transmitters. Figure 2d shows one of the spectrum view from measurements.
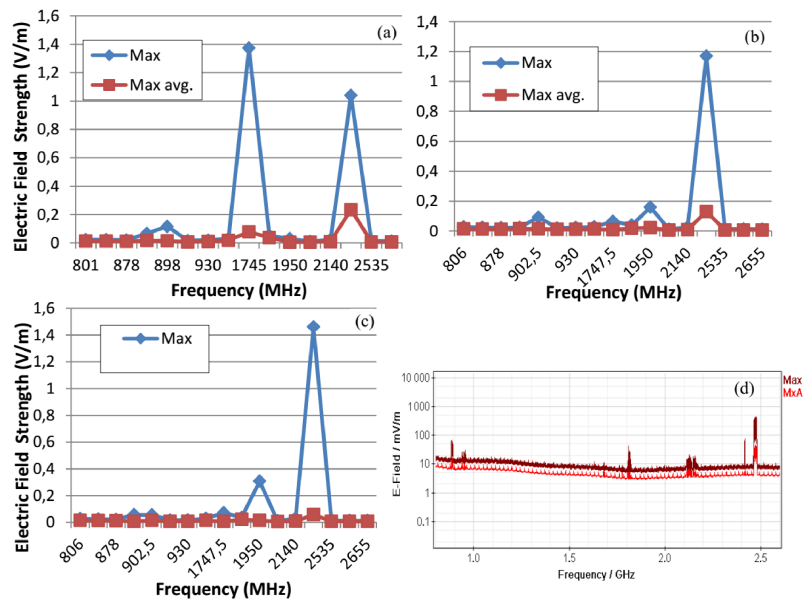

Fig. 2. Electric field strength measurements for different channels and dwellings: (a) for dwelling 1, (b) for dwelling 2, (c) for dwelling 3, (d) one of the spectrum view from measurements.

The measurement results around the Wi-Fi adapter are given in Table III. The results of Table III are plotted in Fig. 3. 


\begin{tabular}{|c|c|c|c|c|c|c|c|c|}
\hline \multirow{3}{*}{ Channel } & \multicolumn{2}{|c|}{ Freq. } & \multicolumn{6}{|c|}{ EFS } \\
\hline & \multirow{2}{*}{\multicolumn{2}{|c|}{$\begin{array}{c}\min . \mid \max . \\
{[\mathrm{MHz}]}\end{array}$}} & \multicolumn{2}{|c|}{ dwelling $\# 1$} & \multicolumn{2}{|c|}{ dwelling \#2 } & \multicolumn{2}{|c|}{ dwelling \#3 } \\
\hline & & & $\max$ & avg. & $\max$. & avg. & $\max$. & avg. \\
\hline 3GPP Band 20 Down; LTE Band 20 Down & 791 & 821 & 0.024 & 0.014 & 0.029 & 0.014 & 0.028 & 0.015 \\
\hline 3GPP Band $20 \mathrm{Up}$; LTE Band $20 \mathrm{Up}$ & 832 & 862 & 0.024 & 0.013 & 0.024 & 0.013 & 0.024 & 0.014 \\
\hline GSM-R Up & 876 & 880 & 0.020 & 0.011 & 0.023 & 0.012 & 0.022 & 0.012 \\
\hline E-GSM; 3GPP Band 8; 3G Class 9; LTE Band 8 Up & 880 & 890 & 0.067 & 0.016 & 0.024 & 0.015 & 0.057 & 0.007 \\
\hline P-GSM; 3GPP Band 8; 3G Class 9; LTE Band 8 Up & 890 & 915 & 0.116 & 0.014 & 0.092 & 0.014 & 0.055 & 0.015 \\
\hline GSM-R Down & 921 & 925 & 0.016 & 0.007 & 0.02 & 0.011 & 0.017 & 0.007 \\
\hline E-GSM Down; 3GPP Band 8 Down; 3G Class 9 Down; LTE Band 8 Down & 925 & 935 & 0.019 & 0.011 & 0.024 & 0.011 & 0.018 & 0.007 \\
\hline E-GSM; 3GPP Band 8; 3G Class 9; LTE Band 8 Down & 935 & 960 & 0.029 & 0.018 & 0.029 & 0.023 & 0.029 & 0.014 \\
\hline DCS 1800; 3GPP Band III; 3G Class 8; LTE Band 3 Up & 1710 & 1785 & 1.374 & 0.078 & 0.065 & 0.009 & 0.072 & 0.007 \\
\hline 3GPP Band III; DCS 1800; 3G Class 8; LTE Band 3 Down & 1805 & 1880 & 0.052 & 0.037 & 0.039 & 0.019 & 0.038 & 0.023 \\
\hline 3GPP Band I Up; LTE Band 1 Up & 1920 & 1980 & 0.03 & 0.006 & 0.159 & 0.023 & 0.307 & 0.014 \\
\hline LTE Band 34 & 2010 & 2025 & 0.01 & 0.006 & 0.011 & 0.005 & 0.01 & 0.006 \\
\hline 3GPP Band I Down; LTE Band 1 Down & 2110 & 2170 & 0.023 & 0.008 & 0.026 & 0.009 & 0.03 & 0.008 \\
\hline WLAN & 2412 & 2484 & 1.040 & 0.234 & 1.170 & 0.129 & 1.460 & 0.058 \\
\hline 3GPP Band 7 Up; 3G Class 13 Up; LTE Band 7 Up & 2500 & 2570 & 0.014 & 0.007 & 0.014 & 0.007 & 0.014 & 0.007 \\
\hline LTE Band 38 & 2570 & 2620 & 0.014 & 0.007 & 0.013 & 0.006 & 0.014 & 0.007 \\
\hline 3GPP Band 7; 3G Class 13; LTE Band 7 Down & 2620 & 2690 & $|-|$ & $\mid-$ & $|0.012|$ & $|0.006|$ & 0.013 & 0.007 \\
\hline
\end{tabular}

TABLE III

The electric field strength $[\mathrm{V} / \mathrm{m}]$ at different distances from the adapter. Wi-Fi modem is $100 \mathrm{~m}$ away.

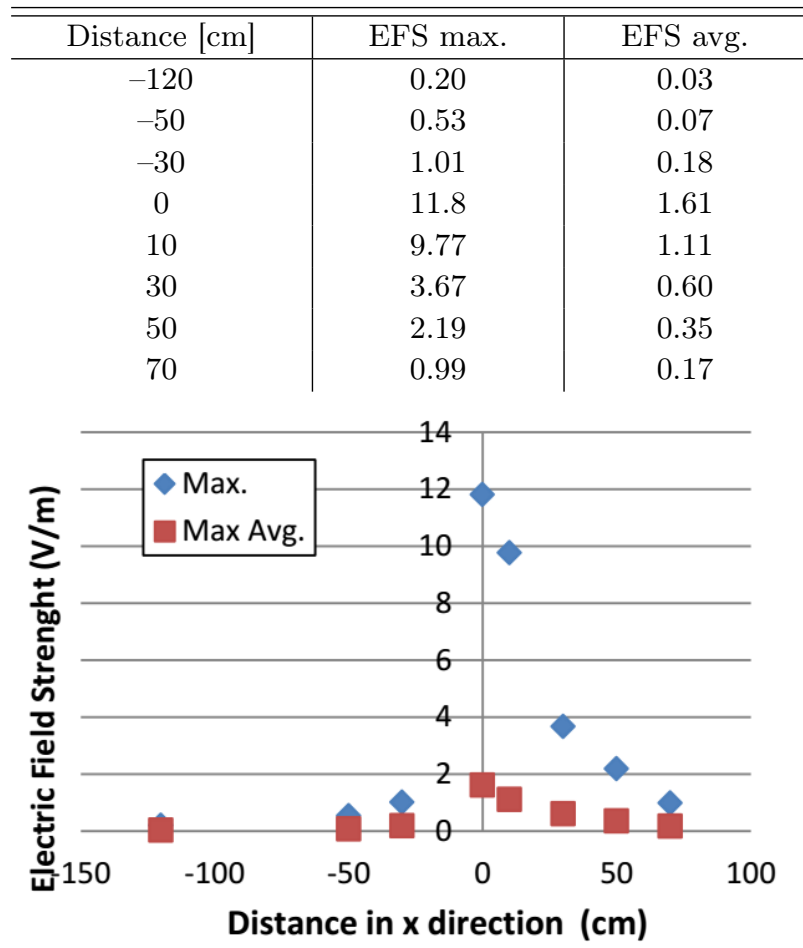

Fig. 3. EFS variation of Wi-Fi adapter with distance.

\section{Conclusions}

In this study, electromagnetic radiation in dwelling is investigated. The all electric field strength values are be- low ICTA limits. Among the channels of GSM, LTE, Wi-Fi EM sources in dwelling, Wi-Fi and LTE (during streaming) are more effective in dwellings. Electric field strength of Wi-Fi adapters are close to ICTA limits. In dwellings, Wi-Fi adapters or high power Wi-Fi repeaters shows more electric field strength compared to GSM technologies. Even thought the instant values are below the SAR values, long term exposure may effect because it is close to upper limit. High power wireless adapters and modems are suggested to keep away from human body.

\section{Acknowledgments}

This study was supported by The Scientific Research Unit of RTE University project no. 2013.102.01.1. The authors wish to thank for the support of RTE University.

\section{References}

[1] C.Z. Zulkifli, H.N. Hassan, W. Ismail, S.N. Semunab, Acta Phys. Pol. A 128, B-86 (2015).

[2] A. Basit, I.M. Qureshi, W. Khan, A.N. Malik, Cognit. Comput. 8, 204 on (2016).

[3] B. Mukanova, T. Mirgalikyzy, Acta Phys. Pol. A 128, B-142 (2015).

[4] A. Pal, R. Tiwari, A. Shukla, Cognit. Comput. 4, 526 (2012).

[5] R. Richman, A.J. Munroe, Y. Siddiqui, Sci. Total Environm. 466, 625 (2014).

[6] B.O. Ayinmode, I.P. Farai, Pacific J. Sci. Technol. 14, 110 (2013).

[7] L. Seyfi, Measurement 46, 3002 (2013).

[8] M. Cansiz, T. Abbasov, M.B. Kurt, A.R. Celik, Measurement 86, 159 (2016). 
[9] N. As, B. Dilek, M.E. Şahin, Y. Karan, Global J. Adv. Pure Appl. Sci. 3, 65 (2014).

[10] D. Urbinello, W. Joseph, A. Huss, L. Verloock, J. Beekhuizen, R. Vermeulen, L. Martens, M. Röösli, Environ Int. 68, 49 (2014).

[11] M.E. Sahin, N. As, Y. Karan, Gazi Univ. J. Sci. 26, 73 (2013).

[12] E. Eyüboğlu, H. Kaya, in: Electrical, Electronics and Computer Engineering, (ELECO) 2010 Nat. Conf., Bursa, (Turkey), 2010, p. 459.

[13] S.I. Henderson, M.J. Bangay, Bioelectromagn. J. 27, 73 (2013)

[14] I. Akkurt, B. Mavi, Sci. Res. Essays 5, 2088 (2010).

[15] Y. Karan, N. As, Turk. J. Electromech. Energy 1, 17 (2016).

[16] M. Elsayed, A.F. Abdelazid, M. Hasna, D. Trinchero, in: Conf. Electromagnetic in Advanced Applications, (ICEAA) Int., Torino, (Italy), 2011, p. 1442.
[17] M.E. Sahin, Y. Karan, N. As, Spectrum Analyzer Measurement Technique for Safety Evaluation on Wireless Communication Systems, URSIGASS, (2011), Istanbul, IEEE Proceeding.

[18] E. Atilgan, Y. Karadana, S. Çömlekçi, SDÜ Teknik Bilimler Dergisi 4 (1), (1) 13 (2014).

[19] A. Ahlbom, Health Phys. 74, 494 (1998).

[20] The Information and Communication Technologies Authority of Turkey (ICTA), Implementing Regulation on the Determination, Control and Supervision of Exposure Limit Values of Electromagnetic Field Intensity According to International Standards, Official Newspaper, 2011, Issue 27912, Revised 2015 with Issue 29497. 